\title{
Revised and extended level scheme of the doubly-odd nucleus ${ }^{188} \mathrm{Ir}$
}

\author{
A. Jungclaus, ${ }^{1}$ V. Modamio, ${ }^{1}$ J. L. Egido, ${ }^{1}$ R. Schwengner, ${ }^{2}$ A. Algora,${ }^{3}$ D. Bazzacco,,${ }^{4}$ D. Escrig, ${ }^{5}$ M. A. Fernandez, ${ }^{1}$ \\ L. M. Fraile, ${ }^{6}$ S. Lenzi, ${ }^{4}$ N. Marginean, ${ }^{4}$ T. Martinez, ${ }^{7}$ D. R. Napoli, ${ }^{7}$ and C. A. Ur ${ }^{4}$ \\ ${ }^{1}$ Departamento de Física Teórica C-XI, Universidad Autónoma de Madrid, E-28049 Madrid, Spain \\ ${ }^{2}$ Institut für Strahlenphysik, Forschungszentrum Dresden-Rossendorf, D-01314 Dresden, Germany \\ ${ }^{3}$ IFIC, CSIC-Univ.Valencia, E-46071 Valencia, Spain \\ ${ }^{4}$ Istituto Nazionale di Fisica Nucleare, Sezione di Padova, I-35131 Padova, Italy \\ ${ }^{5}$ Instituto de Estructura de la Materia, CSIC, E-28040 Madrid, Spain \\ ${ }^{6}$ CERN, ISOLDE, CH-1211 Geneva 23, Switzerland \\ ${ }^{7}$ Istituto Nazionale di Fisica Nucleare, Laboratori Nazionali di Legnaro, I-35020 Legnaro, Italy \\ (Received 9 April 2006; revised manuscript received 14 September 2007; published 25 February 2008)
}

\begin{abstract}
High-spin states in the doubly odd $Z=77$ nucleus ${ }^{188} \mathrm{Ir}$ were studied using the reaction ${ }^{186} \mathrm{~W}\left({ }^{7} \mathrm{Li}, 5 n\right)$ at $59 \mathrm{MeV}$ and the GASP spectrometer for $\gamma$-ray detection. The level structures recently suggested to be built on the known 4.1(3) ms isomeric state of this nucleus have been considerably revised and extended and an isomer with a lifetime of 17.7(2) ns has been identified within the main decay sequence. In addition two rotational bands built on low spin states below the ms isomer have been observed for the first time. The basic features of the excitation scheme of ${ }^{188}$ Ir are discussed within the Hartree-Fock-Bogoliubov theory within the Lipkin-Nogami approach with the finite-range density-dependent Gogny force.
\end{abstract}

DOI: 10.1103/PhysRevC.77.024310 PACS number(s): 23.20.Lv, 23.20.En, 27.70.+q, 21.60.Jz

\section{INTRODUCTION}

In a recent article, Balabanski et al. presented the first extensive work about the high-spin structure of the doubly odd $Z=77$ nucleus ${ }^{188} \operatorname{Ir}$ [1]. The level scheme proposed in that work comprises two regular bands as well as a number of irregularly spaced levels of single-particle structure. The two regular bands, consisting of $\Delta I=1$ sequences with parallel crossover transitions, have both been assigned the $\pi h_{9 / 2} \otimes \nu i_{13 / 2}$ configuration and suggested to be first candidates for the observation of a chiral doublet in the mass $A \approx 190$ region. Because for the higher-lying band only two levels have been observed for each signature the intention of our present work was to extend the two bands to higher spin to put this assignment on a safer footing. Such an extension would be crucial to prove the nature of the observed bands on the basis of established criteria such as a small energy staggering, intense $M 1$ and $E 2$ linking transitions between levels of the two bands, and similar $B(M 1) / B(E 2)$ ratios within the two partner bands [2-4]. Considering that these two bands in ${ }^{188} \mathrm{Ir}$ are the so far only candidates for chiral doublets in the $A \approx 190$ mass region and the fact that in theoretical studies of this nucleus the $\pi i_{13 / 2} \otimes \nu i_{13 / 2}$ rather than the $\pi h_{9 / 2} \otimes \nu i_{13 / 2}$ configuration has been proposed as a candidate for chiral doublets [5], such a proof would be highly desirable. In addition, we wanted to carefully check the entire level scheme because some of its aspects seemed to require further clarification. One example is the fact that of the four $16^{-}$states observed within $186 \mathrm{keV}$ the highest one collects nearly all the intensity flux, whereas the yrast state of this spin is only very weakly populated [1].

\section{EXPERIMENTAL DETAILS AND RESULTS}

High-spin states in the nucleus ${ }^{188} \mathrm{Ir}$ were populated using the reaction ${ }^{186} \mathrm{~W}\left({ }^{7} \mathrm{Li}, 5 n\right)$. The beam with an energy of
$59 \mathrm{MeV}$ was delivered by the XTU tandem accelerator of the Laboratori Nazionali di Legnaro and directed onto a thick target of $12 \mathrm{mg} / \mathrm{cm}^{2}{ }^{186} \mathrm{~W}$. The $\gamma$ radiation was detected in the 40 Compton suppressed Ge detectors of the GASP array and the 80-element BGO inner ball. The trigger condition required at least two Ge detectors and one BGO firing in coincidence. The time distribution of the $\gamma$ rays detected in the Ge detectors was measured in a time window of $200 \mathrm{~ns}$ with respect to the prompt time signal of the BGO. From the observed coincident $\gamma$ rays $E_{\gamma}-E_{\gamma}$ matrices as well as $E_{\gamma}-E_{\gamma}-E_{\gamma}$ cubes were constructed both with and without applying a prompt time condition. The latter has been accomplished applying two-dimensional banana gates on the prompt region in the energy-time matrices separately for all $40 \mathrm{Ge}$ detectors. The construction of the level scheme of ${ }^{188} \mathrm{Ir}$ to be discussed in the next section is mainly based on the analysis of the prompt cube.

To determine $\gamma$-ray multipolarities from the analysis of the directional correlation ratios from oriented states (DCO), $\gamma-\gamma$ events with one $\gamma$ ray detected in one of the 12 detectors positioned at angles of $31.7^{\circ}, 36^{\circ}, 144^{\circ}$, and $148.3^{\circ}$ (weighted averages $35^{\circ}$ or $145^{\circ}$, respectively) and the other one detected in one of the eight detectors at $72^{\circ}$ and $108^{\circ}$ relative to the beam direction were sorted into a coincidence matrix. Using a coincidence gate on a stretched quadrupole transition $\gamma_{\text {gate }}$, the DCO ratio

$$
\begin{aligned}
R_{\mathrm{DCO}}= & \frac{I_{\gamma}\left(\gamma \text { observed at } \Theta_{1} ; \gamma_{\text {gate }} \text { at } \Theta_{2}\right)}{I_{\gamma}\left(\gamma \text { observed at } \Theta_{2} ; \gamma_{\text {gate }} \text { at } \Theta_{1}\right)} \\
& \times \frac{\epsilon_{\Theta_{1}}(\gamma) \epsilon_{\Theta_{2}}\left(\gamma_{\text {gate }}\right)}{\epsilon_{\Theta_{1}}\left(\gamma_{\text {gate }}\right) \epsilon_{\Theta_{2}}(\gamma)}
\end{aligned}
$$

is $\approx 1$ for stretched quadrupole and $\approx 0.6$ for pure dipole transitions $\left(\Theta_{1}=35^{\circ}, 145^{\circ}, \Theta_{2}=72^{\circ}, 108^{\circ}\right) . \epsilon_{\Theta}(\gamma)$ is the relative efficiency at the energy $E_{\gamma}$ of the detectors positioned at an angle $\Theta$ with respect to the beam. 


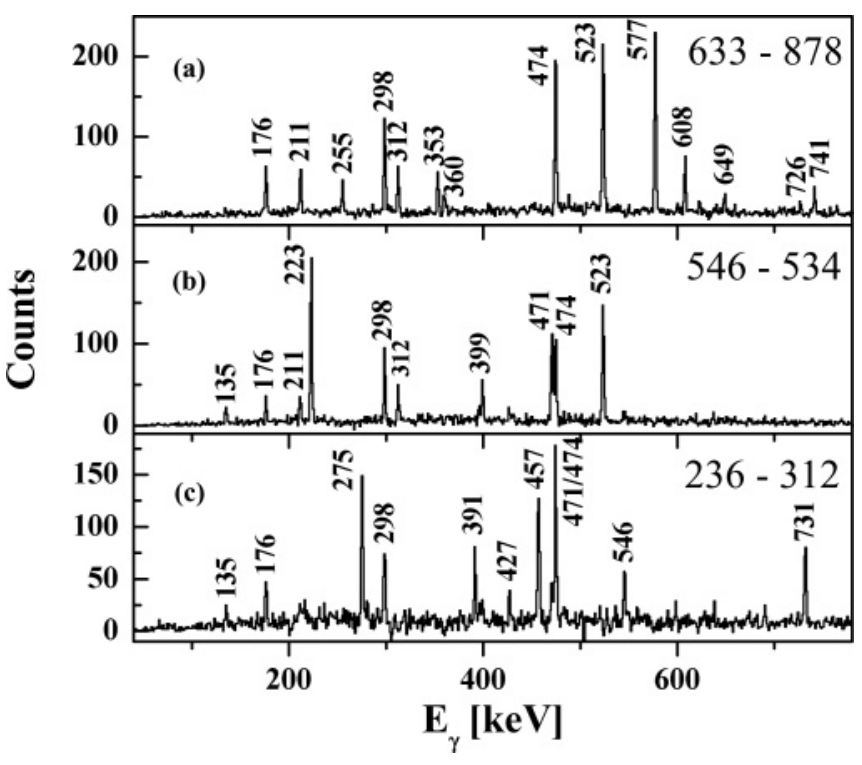

FIG. 1. Double-gated coincidence spectra in ${ }^{188} \mathrm{Ir}$ with gates on (a) the 633- and 878-keV, (b) the 546- and 534-keV, and (c) the 236and $312-\mathrm{keV}$ transitions.

In the following subsections, we will present the level scheme of ${ }^{188} \mathrm{Ir}$ deduced in the present work, discuss the spin assignments for some excited states, and determine the lifetime of the newly identified isomeric state.

\section{A. The level scheme of ${ }^{188}$ Ir}

The low-spin part of the level scheme of ${ }^{188} \mathrm{Ir}$ has been established already more than 20 years ago by Kreiner et al. [6] in an experiment employing the ${ }^{187} \operatorname{Re}(\alpha, 3 n)$ reaction. In that work, the highest observed state had an excitation energy of $924 \mathrm{keV}$ and a spin assignment of $I^{\pi}=(8-11)^{-}$. All $\gamma$ rays observed by Kreiner et al. "were found to have both significant delayed, and to a lesser extent prompt components." The half-life of the observed delayed components is $T_{1 / 2}=$ 4.1(3) $\mathrm{ms}$ in all cases. The authors concluded from this observation first that most of the population of ${ }^{188} \mathrm{Ir}$ is trapped at the already known $T_{1 / 2}=4.1(3) \mathrm{ms}[7,8]$ isomeric state before it decays to the ground state and second that "none of the observed lines depopulated directly the isomer suggesting its decay by a highly converted low-energy transition." Very recently, Balabanski et al. [1] presented a level scheme comprising high-spin states above the isomer obtained in a measurement employing the same reaction as in the present work, namely ${ }^{186} \mathrm{~W}\left({ }^{7} \mathrm{Li}, 5 n\right)$. However, the inspection of some double gated coincidence spectra directly reveal inconsistencies of the level scheme proposed in Ref. [1]. The coincidence spectrum with gates on both the 633- and 878-keV transitions is shown in Fig. 1(a). According to the level scheme of Ref. [1] peaks at $306,399,503,353$, and $360 \mathrm{keV}$ corresponding to $\gamma$ transition above the $878-\mathrm{keV}$ transition should be visible in this spectrum, in addition to the transitions below the $13^{-}$ state (the spins in this paragraph refer to the values quoted in Ref. [1]). However, only the 353- and 360-keV peaks are there, but in addition strong transitions at 577, 608, and
$255 \mathrm{keV}$ are observed in this spectrum. This observation leads to the suspicion that the $878.7 \mathrm{keV} 17^{+} \rightarrow 15^{-}$transition is probably not parallel to the 575.7- and 304.4-keV $\gamma$ rays. Note that the energy sum of the latter two $\gamma$ rays is $880.1 \mathrm{keV}$, $1.4 \mathrm{keV}$ larger than the quoted energy of the $878.7-\mathrm{keV}$ transition. A second problem occurs when looking at the double-gated spectrum with gates on the 546- and 534-keV transitions shown in Fig. 1(b). The strongest line in this spectrum is the one at $223 \mathrm{keV}$, which according to the level scheme of Ref. [1] should not be there because the 223-keV transition is parallel to the one at $546 \mathrm{keV}$. Concerning the higher-lying chiral partner band labeled Band 2 in Ref. [1] a coincidence spectrum with gate on the $236 \mathrm{keV}\left(15^{-}\right) \rightarrow$ $\left(14^{-}\right) M 1$ transition within that band and the $312 \mathrm{keV} 12^{-} \rightarrow$ $11^{-}$yrast transition is shown in Fig. 1(c). According to the level scheme proposed in Ref. [1] the 266- and 523-keV transitions feeding the $\left(15^{-}\right)$level should be seen in this spectrum in contrast to the experimental observation. Instead, transitions with $391,427,471$, and $546 \mathrm{keV}$ are observed.

A careful analysis of all double-gated coincidence spectra led to the new revised and extended level scheme built on the isomeric state presented in Fig. 2. The main differences between this new level scheme and the version of Ref. [1] are (i) the placement of the $88-\mathrm{keV}$ transition between the 304- and 633-keV transitions within the main decay sequence, (ii) the identification of the two $15^{-}$states shown on the leftand right-hand sides of the level scheme in Ref. [1] as being one state, and (iii) the identification of two close-lying states at $\Delta+2597 \mathrm{keV}$ and $\Delta+2599 \mathrm{keV}$ decaying via the $878-$ and $575-\mathrm{keV}$ transitions, respectively. In addition to these differences, the level scheme presented in this work comprises many additional levels and $\gamma$ rays not observed in Ref. [1]. The reason for the placement of the $88-\mathrm{keV}$ transition directly above the 474-523-633-keV sequence was the observation that there is a severe reduction in observed intensities for these three transitions in comparison to the 304-, 575-, and $570-\mathrm{keV}$ transitions in, for example, the gate on the $956-\mathrm{keV}$ doublet in the prompt $E_{\gamma}-E_{\gamma}$ matrix.

This gate is shown in Fig. 3(a). The same coincidence spectrum created from the $E_{\gamma}-E_{\gamma}$ matrix without time gate is shown in Fig. 3(b). In this spectrum, the intensity balance is correct. Note that a $955-\mathrm{keV}$ transition is also observed in the strongest reaction channel, namely ${ }^{187} \mathrm{Ir}$. In this nucleus the most intense $\gamma$ rays observed in prompt coincidence with this transition have energies of 267, 504, and $489 \mathrm{keV}$ [9] and are labeled with a $\mathrm{C}$ in Fig. 3(a). Due to this contamination the intensity of the $266-\mathrm{keV}$ transition in the prompt spectrum seems to be too high compared, for example, to the $633-\mathrm{keV}$ $\gamma$ ray, the second decay branch of the $\left(15^{-}\right)$state at $1631 \mathrm{keV}$. From the observed differences in intensities in the two spectra of Fig. 3, we can deduce that the 474-523-633-keV sequence is below an isomer with a lifetime of at least some ns and the low-energy $88-\mathrm{keV}$ transition is a good candidate to depopulate such an isomer. This placement is confirmed by the many indirect connections between the states above and below this isomer, for example, the fitting energy sums of the 633-, 88-, 304-, 575-, and 306-keV, respectively, 534-, 223-, 546-, $471-$, and $135-\mathrm{keV}$ cascades. The determination of the lifetime of this newly identified isomeric state will be discussed in 


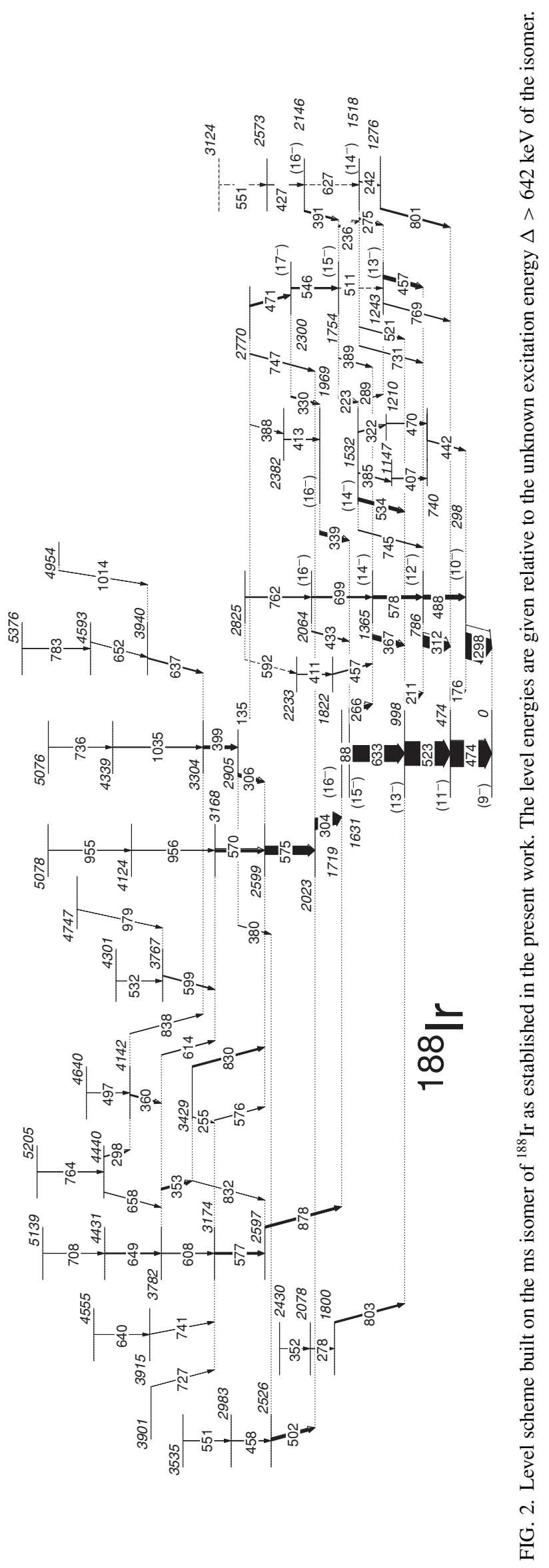




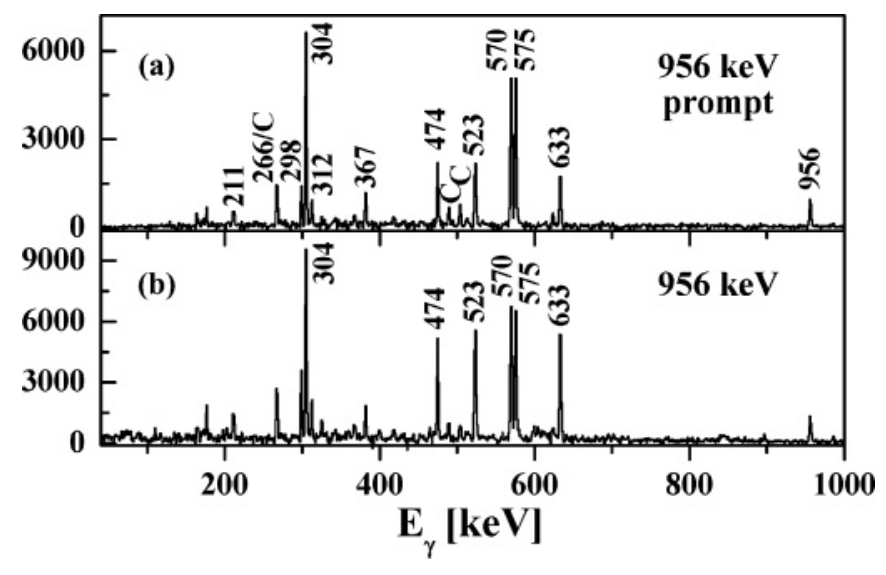

FIG. 3. Coincidence spectra with gate on the $956 \mathrm{keV}$ doublet with (a) a prompt time window and (b) without time condition. The lines labeled with a $\mathrm{C}$ are the strongest transitions in prompt coincidence with a $955 \mathrm{keV} \gamma$ ray in the neighboring isotope ${ }^{187} \mathrm{Ir}$, the strongest reaction channel [9].

subsection IIC. With respect to the structure named "Band 2" in Ref. [1], we found no evidence for the placement of the 266-, 502-, and (522)-keV transitions. Instead, we place the 391- and 546-keV transitions on top of the $\Delta+1754-\mathrm{keV}$ level. In the double gate on the 236- and 391-keV $\gamma$ rays in Fig. 4(a) one more peak is visible at $427 \mathrm{keV}$ and we place this transition and tentatively another one with $551 \mathrm{keV}$ on top of the 391-keV transition. Note that strong decay branches of the $\left(14^{-}\right)$state at $\Delta+1518 \mathrm{keV}$ to the $\left(12^{-}\right)$and $\left(13^{-}\right)$ yrast states (via 731-, respectively, 521-keV transitions) as well as a two-step decay $(242-801 \mathrm{keV})$ to the $\left(11^{-}\right)$level are

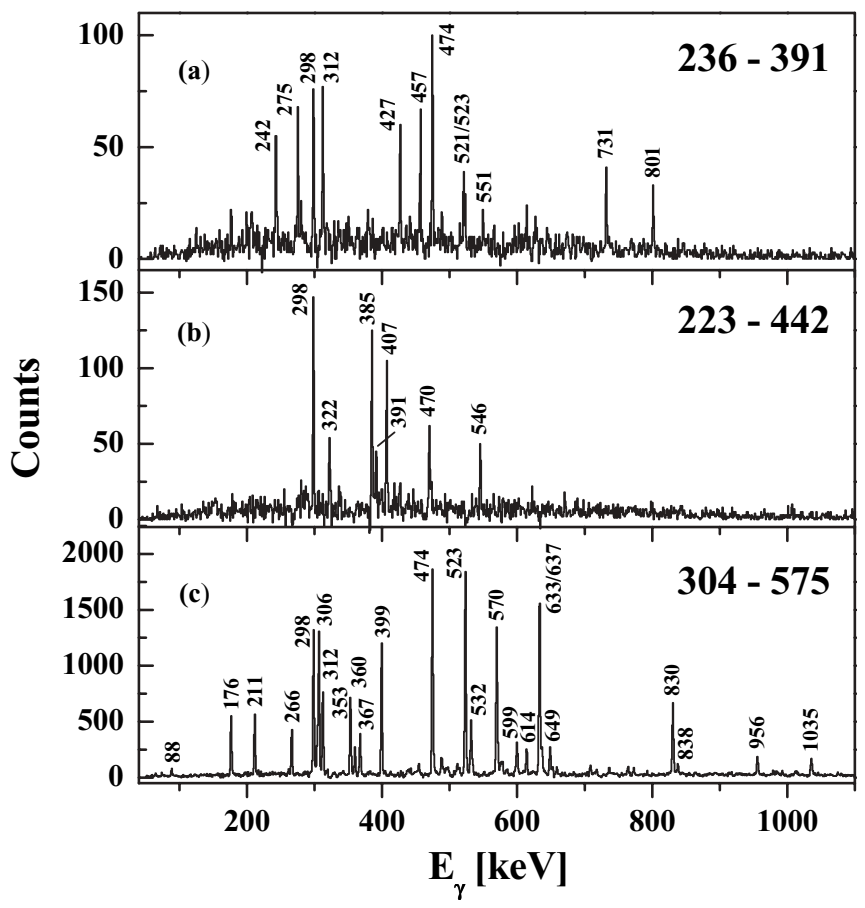

FIG. 4. Double-gated coincidence spectra in ${ }^{188} \mathrm{Ir}$ with gates on (a) the 236- and 391-keV, (b) the 223- and 442-keV, and (c) the 304and $575-\mathrm{keV}$ transitions. observed [see Fig. 4(a)]. The spectrum with gates on the 223and 442-keV transitions shown in Fig. 4(b) illustrates the two parallel decay branches of the third $\left(14^{-}\right)$level at $\Delta+1532 \mathrm{keV}$ as well as the feeding of the $\left(15^{-}\right)$state of Band 2 via the 471 to $546-\mathrm{keV}$ cascade.

The $\Delta+2599-\mathrm{keV}$ level within the main decay sequence is fed by three different branches as shown in the level scheme and reflected by the observation of three strong $\gamma$-transitions at 306,570 , and $830 \mathrm{keV}$ in the double-gated spectrum with gates on the 304- and 575-keV transitions [see Fig. 4(c)]. Because the $570-\mathrm{keV}$ line is clearly observed in this spectrum, this transition cannot be placed parallel to the 304-keV transition as suggested in Ref. [1]. On top of the 570-keV transition we placed two $\gamma$ rays with $956 \mathrm{keV}$ because the $956-\mathrm{keV}$ line is clearly observed in self-coincidence as shown in Fig. 3. On top of the $306-\mathrm{keV}$ transition we place the $399-\mathrm{keV} \gamma$ ray (as done in Ref. [1]) and the intermediate state at $\Delta+2905 \mathrm{keV}$ decays both to the yrast sequence as well as via the $135-\mathrm{keV}$ transition to the structures on the right-hand side of Fig. 2. Information on all transitions placed in the high-spin level scheme above the ms isomeric state in ${ }^{188} \mathrm{Ir}$ are summarized in Table I.

In a next step we tried to extend the low-spin level scheme based on the ground state of ${ }^{188} \mathrm{Ir}$ presented by Kreiner et al. [6]. We created the sum of double-gated spectra with gates on two of the three strong transitions with 150, 143, and $138 \mathrm{keV}$, respectively. This sum spectrum is plotted in Fig. 5(a). One clearly observes many more peaks in addition to the ones expected from the known level scheme [6]. A careful analysis of the coincidence data led to a considerable extension of the low-spin scheme as shown in Fig. 6. The information concerning all newly placed $\gamma$ transitions in this scheme is summarized in Table II. We found two relatively strong transitions of 160 and $173 \mathrm{keV}$ feeding the 642-keV state. A regular rotational band consisting of 423-, 516-, 599-, $675-$, and $653-\mathrm{keV}$ transitions is illustrated by the coincidence

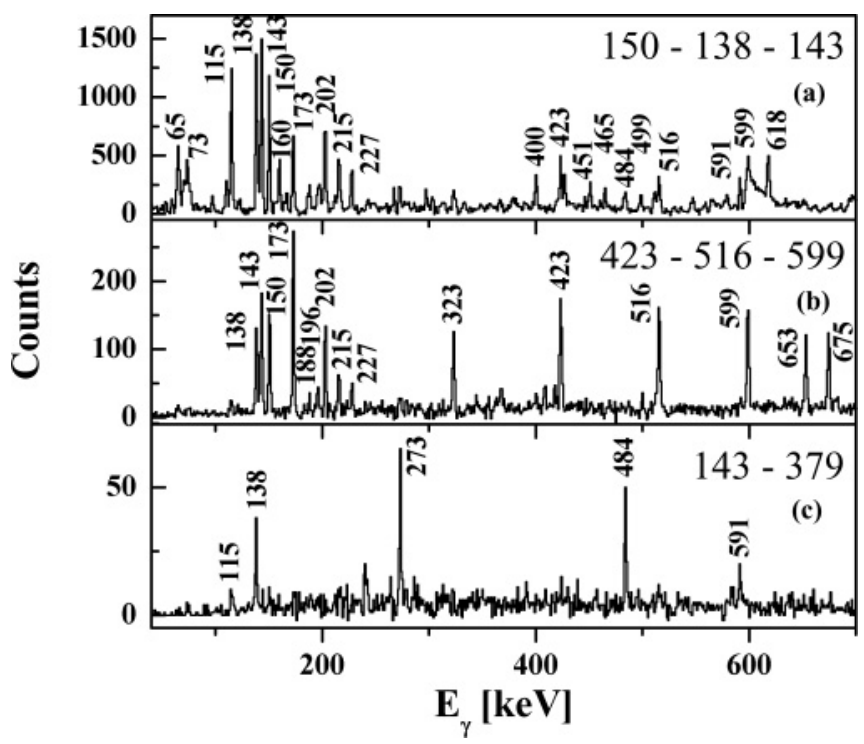

FIG. 5. Double-gated coincidence spectra in ${ }^{188} \mathrm{Ir}$ with gates on twofold combinations of (a) the 150-, 138-, and 143-keV; (b) the 423-, 516-, and 598-keV; and (c) the 143- and 379-keV transitions. 
TABLE I. Energies, intensities, DCO ratios, initial-state excitation energy, and initial- and final-state spins of $\gamma$ transitions placed in the level scheme built on the ms isomeric state in ${ }^{188}$ Ir. The intensities are not corrected for internal conversion.

\begin{tabular}{|c|c|c|c|c|c|}
\hline$E_{\gamma}(\mathrm{keV})^{\mathrm{a}}$ & $I_{\gamma}$ & $R_{\mathrm{DCO}}$ & $E_{i}(\mathrm{keV})$ & $I_{i}^{\pi}$ & $I_{f}^{\pi}$ \\
\hline 88.3 & $69(4)$ & $1.36(34)$ & 1719 & $\left(16^{-}\right)$ & $\left(15^{-}\right)$ \\
\hline 134.9 & $24(1)$ & $0.99(22)$ & 2905 & & \\
\hline 176.2 & $243(6)$ & & 474 & $\left(11^{-}\right)$ & $\left(10^{-}\right)$ \\
\hline 211.2 & 161(4) & $0.74(6)$ & 998 & $\left(13^{-}\right)$ & $\left(12^{-}\right)$ \\
\hline 222.5 & $94(6)$ & $0.97(6)$ & 1754 & $\left(15^{-}\right)$ & $\left(14^{-}\right)$ \\
\hline 235.8 & $39(1)$ & $0.58(13)$ & 1754 & $\left(15^{-}\right)$ & $\left(14^{-}\right)$ \\
\hline 241.9 & $29(2)$ & & 1518 & $\left(14^{-}\right)$ & \\
\hline 255.0 & $7(1)$ & & 3429 & & \\
\hline 266.2 & $97(4)$ & & 1631 & $\left(15^{-}\right)$ & $\left(14^{-}\right)$ \\
\hline 275.2 & $21(1)$ & $0.51(13)$ & 1518 & $\left(14^{-}\right)$ & $\left(13^{-}\right)$ \\
\hline 277.4 & $30(1)$ & & 2078 & & \\
\hline 289.3 & $12(1)$ & & 1532 & $\left(14^{-}\right)$ & $\left(13^{-}\right)$ \\
\hline \multirow[t]{2}{*}{$298.3^{b}$} & $218(12)$ & $0.89(2)$ & 298 & $\left(10^{-}\right)$ & $\left(9^{-}\right)$ \\
\hline & & & 4440 & & \\
\hline 304.2 & $605(12)$ & $0.70(2)$ & 2023 & & $\left(16^{-}\right)$ \\
\hline 306.2 & $90(3)$ & $0.73(3)$ & 2905 & & \\
\hline 312.0 & $403(10)$ & $0.86(7)$ & 786 & $\left(12^{-}\right)$ & $\left(11^{-}\right)$ \\
\hline 322.2 & $17(1)$ & & 1532 & & \\
\hline 330.3 & $82(3)$ & $0.81(1)$ & 2300 & $\left(17^{-}\right)$ & $\left(16^{-}\right)$ \\
\hline 338.8 & $94(3)$ & $0.70(3)$ & 1969 & $\left(16^{-}\right)$ & $\left(15^{-}\right)$ \\
\hline 351.7 & $15(1)$ & & 2430 & & \\
\hline 353.0 & $63(3)$ & $1.02(9)$ & 3782 & & \\
\hline 359.7 & $49(2)$ & & 4142 & & \\
\hline 367.1 & $168(4)$ & $0.81(4)$ & 1365 & $\left(14^{-}\right)$ & $\left(13^{-}\right)$ \\
\hline 379.5 & $14(4)$ & & 2905 & & \\
\hline 384.6 & $14(1)$ & & 1532 & $\left(14^{-}\right)$ & \\
\hline \multirow[t]{2}{*}{$389.1^{\mathrm{b}}$} & $21(1)$ & & 1754 & $\left(15^{-}\right)$ & $\left(14^{-}\right)$ \\
\hline & & & 2770 & & \\
\hline 391.0 & $34(2)$ & & 2146 & $\left(16^{-}\right)$ & $\left(15^{-}\right)$ \\
\hline 399.0 & $133(4)$ & $0.89(8)$ & 3304 & & \\
\hline 406.7 & $35(1)$ & & 1147 & & \\
\hline 410.9 & $19(1)$ & & 2233 & & \\
\hline 412.7 & $24(1)$ & & 2382 & & \\
\hline 427.0 & $73(3)$ & & 2573 & & $\left(16^{-}\right)$ \\
\hline 432.8 & $46(2)$ & & 2064 & $\left(16^{-}\right)$ & $\left(15^{-}\right)$ \\
\hline 441.8 & $100(4)$ & & 740 & & \\
\hline \multirow[t]{2}{*}{$456.7^{\mathrm{b}}$} & $106(4)$ & $0.92(7)$ & 1243 & $\left(13^{-}\right)$ & $\left(12^{-}\right)$ \\
\hline & & & 1821 & & $\left(14^{-}\right)$ \\
\hline 458.4 & $24(2)$ & & 2983 & & \\
\hline 469.8 & $64(2)$ & & 1210 & & \\
\hline 470.6 & $51(2)$ & $0.99(9)$ & 2770 & & $\left(17^{-}\right)$ \\
\hline 474.4 & $1383(86)$ & $1.02(3)$ & 474 & $\left(11^{-}\right)$ & $\left(9^{-}\right)$ \\
\hline 488.1 & 197(6) & $1.30(18)$ & 786 & $\left(12^{-}\right)$ & $\left(10^{-}\right)$ \\
\hline 497.3 & $7(1)$ & & 4640 & & \\
\hline 502.3 & $77(4)$ & $1.14(24)$ & 2526 & & \\
\hline 521.0 & 11(3) & & 1518 & & \\
\hline 523.2 & $1000(19)$ & $0.98(3)$ & 998 & $\left(13^{-}\right)$ & $\left(11^{-}\right)$ \\
\hline 531.5 & $41(4)$ & & 4301 & & \\
\hline 533.9 & $98(3)$ & $1.09(12)$ & 1532 & $\left(14^{-}\right)$ & $\left(13^{-}\right)$ \\
\hline 545.7 & $52(2)$ & $0.81(9)$ & 2300 & $\left(17^{-}\right)$ & $\left(15^{-}\right)$ \\
\hline 551.4 & 11(3) & & 3124 & & \\
\hline 552.1 & $52(2)$ & & 3535 & & \\
\hline 569.5 & $113(3)$ & $1.00(9)$ & 3168 & & \\
\hline 575.4 & 408(9) & $0.99(5)$ & 2599 & & \\
\hline
\end{tabular}

TABLE I. (Continued.)

\begin{tabular}{|c|c|c|c|c|c|}
\hline$E_{\gamma}(\mathrm{keV})^{\mathrm{a}}$ & $I_{\gamma}$ & $R_{\mathrm{DCO}}$ & $E_{i}(\mathrm{keV})$ & $I_{i}^{\pi}$ & $I_{f}^{\pi}$ \\
\hline \multirow[t]{2}{*}{$577.1^{\mathrm{b}}$} & \multirow[t]{2}{*}{$70(4)$} & & 3174 & & \\
\hline & & & 3174 & & \\
\hline 578.0 & $152(4)$ & & 1365 & & \\
\hline 591.5 & $37(2)$ & & 2825 & & \\
\hline 599.3 & $30(5)$ & $1.04(15)$ & 3767 & & \\
\hline 607.7 & $5(1)$ & & 3782 & & \\
\hline 614.0 & $31(4)$ & & 3782 & & \\
\hline 633.0 & $879(18)$ & $1.01(7)$ & 1631 & \multirow[t]{8}{*}{$\left(15^{-}\right)$} & \multirow[t]{8}{*}{$\left(13^{-}\right)$} \\
\hline 636.5 & $69(4)$ & & 3940 & & \\
\hline 648.5 & $29(3)$ & & 4431 & & \\
\hline 652.4 & $11(2)$ & & 4593 & & \\
\hline 658.2 & $5(1)$ & & 4440 & & \\
\hline 699.1 & $42(2)$ & & 2064 & & \\
\hline 708.8 & $13(3)$ & & 5139 & & \\
\hline 726.4 & $6(1)$ & & 3902 & & \\
\hline 731.4 & $15(3)$ & & 1518 & \multirow[t]{3}{*}{$\left(14^{-}\right)$} & \multirow[t]{3}{*}{$\left(12^{-}\right)$} \\
\hline 736.3 & $7(1)$ & & 5076 & & \\
\hline 741.0 & $12(1)$ & & 3915 & & \\
\hline 745.4 & $12(1)$ & & 1532 & \multirow[t]{6}{*}{$\left(14^{-}\right)$} & \multirow[t]{4}{*}{$\left(12^{-}\right)$} \\
\hline 747.0 & $38(3)$ & \multirow[t]{6}{*}{$1.05(2)$} & 2770 & & \\
\hline 761.5 & $24(1)$ & & 2825 & & \\
\hline 764.3 & $16(2)$ & & 5205 & & \\
\hline 768.7 & $16(2)$ & & 1243 & & $\left(11^{-}\right)$ \\
\hline 801.3 & $37(2)$ & & 1276 & & $(11-)$ \\
\hline 802.8 & $58(3)$ & & 1800 & $\left(13^{-}\right)$ & \\
\hline 830.4 & $66(4)$ & \multirow[t]{3}{*}{$0.66(7)$} & 3429 & & \\
\hline 831.6 & $3(1)$ & & 3429 & & \\
\hline 837.6 & $26(3)$ & & 4142 & & \\
\hline 878.0 & 104(3) & $0.94(12)$ & 2597 & & $\left(16^{-}\right)$ \\
\hline \multirow[t]{2}{*}{$955.5^{\mathrm{b}}$} & \multirow[t]{2}{*}{$12(1)$} & \multirow[t]{2}{*}{$1.01(16)$} & 4124 & & \\
\hline & & & 5078 & & \\
\hline 1013.7 & $16(2)$ & & 4954 & & \\
\hline 1034.9 & $9(1)$ & $0.89(25)$ & 4339 & & \\
\hline
\end{tabular}

${ }^{\text {aThe uncertainty in the determination of the } \gamma \text {-ray energy is typically }}$ $0.1 \mathrm{keV}$ for transitions with $I_{\gamma} \geqslant 50$ and $0.2 \mathrm{keV}$ for weaker transitions.

${ }^{\mathrm{b}}$ Unresolved doublet.

spectrum shown in Fig. 5(b), which is the sum of double-gated spectra with gates on the 423-, 516-, and 599-keV transitions in the prompt cube. A second much weaker regular structure consisting of transitions of 273, 379, 484, 591, 677, and $732 \mathrm{keV}$ is illustrated in Fig. 5(c), which shows a spectrum with gates on the 143- and 379-keV transitions in the prompt cube. In this spectrum, the $138-\mathrm{keV}$ transition is clearly observed, whereas the $150-\mathrm{keV}$ line is not in coincidence with the 143and $379-\mathrm{keV}$ transitions, indicating that this rotational band is built on the 492-keV level. A further careful analysis revealed that the placement of the 66- and $215-\mathrm{keV}$ transitions on top of the 642-keV state in Ref. [6] is most probably wrong. We tentatively place the $215-\mathrm{keV}$ transition to populate the $354-\mathrm{keV}$ state. By comparing the coincidence intensities in gates created from the prompt and the nongated cube we could also conclude that the $642-\mathrm{keV}$ state has a lifetime of some ns. Spin assignments have been proposed in Ref. [6] based on conversion electron measurements. The 138- and 150-keV 
TABLE II. Energies, intensities, initial-state excitation energy, and initial- and final-state spins of $\gamma$-transitions placed in the level scheme built on the ground state of ${ }^{188}$ Ir. The intensities are not corrected for internal conversion.

\begin{tabular}{lcccc}
\hline \hline$E_{\gamma}(\mathrm{keV})$ & $I_{\gamma}$ & $E_{i}(\mathrm{keV})$ & $I_{i}^{\pi}$ & $I_{f}^{\pi}$ \\
\hline 150.2 & $1000(35)$ & 642 & $\left(8^{+}\right)$ & $\left(6^{+}\right)$ \\
159.7 & $200(22)$ & 802 & & \\
172.8 & $337(30)$ & 815 & $\left(9^{+}\right)$ & $\left(8^{+}\right)$ \\
196.2 & $96(12)$ & 1238 & $\left(11^{+}\right)$ & $\left(10^{+}\right)$ \\
227.4 & $169(16)$ & 1042 & $\left(10^{+}\right)$ & $\left(9^{+}\right)$ \\
272.5 & $256(24)$ & 765 & $\left(8^{+}\right)$ & $\left(6^{+}\right)$ \\
302.4 & $46(11)$ & 1540 & $\left(12^{+}\right)$ & $\left(11^{+}\right)$ \\
322.5 & $98(13)$ & 815 & $\left(9^{+}\right)$ & $\left(6^{+}\right)$ \\
378.5 & $107(12)$ & 1143 & $\left(10^{+}\right)$ & $\left(8^{+}\right)$ \\
400.4 & $176(17)$ & 1042 & $\left(10^{+}\right)$ & $\left(8^{+}\right)$ \\
423.2 & $261(20)$ & 1238 & $\left(11^{+}\right)$ & $\left(9^{+}\right)$ \\
450.9 & $123(23)$ & 1253 & & \\
464.7 & $44(9)$ & 1718 & & \\
483.8 & $137(16)$ & 1627 & $\left(12^{+}\right)$ & $\left(10^{+}\right)$ \\
498.5 & $118(33)$ & 1540 & $\left(12^{+}\right)$ & $\left(10^{+}\right)$ \\
515.6 & $180(30)$ & 1754 & $\left(13^{+}\right)$ & $\left(11^{+}\right)$ \\
580.5 & $62(21)$ & 2121 & $\left(14^{+}\right)$ & $\left(12^{+}\right)$ \\
591.3 & $191(23)$ & 2218 & $\left(14^{+}\right)$ & $\left(12^{+}\right)$ \\
598.9 & $123(17)$ & 2352 & $\left(15^{+}\right)$ & $\left(13^{+}\right)$ \\
617.6 & $263(30)$ & 1420 & & \\
652.7 & $44(8)$ & 3680 & $\left(19^{+}\right)$ & $\left(17^{+}\right)$ \\
674.6 & $44(7)$ & 3027 & $\left(17^{+}\right)$ & $\left(15^{+}\right)$ \\
676.6 & $82(9)$ & 2895 & $\left(16^{+}\right)$ & $\left(14^{+}\right)$ \\
732.3 & & 3627 & & \\
\hline \hline & & & & \\
\hline
\end{tabular}

transitions had been assigned $E 2$ nature leading to a $\left(6^{+}\right)$ assignment for the $492-\mathrm{keV}$ state and an $\left(8^{+}\right)$assignment for the $642-\mathrm{keV}$ state. Considering the fact that the regular band built on the $815-\mathrm{keV}$ level collects clearly more intensity than the one based on the 492-keV state we tentatively assign spin $\left(9^{+}\right)$to the $815-\mathrm{keV}$ bandhead.

\section{B. Discussion of spin assignments}

The ms isomeric state had been assigned spin and parity $9^{-}$in Ref. [1] based on (i) the available information about low-spin states [6], (ii) the amplitude and phase of the energy staggering of the yrast sequence in comparison to the even-even ${ }^{182,184,186} \mathrm{Ir}$ isotopes, (iii) the systematics of excitation energies of the $\pi h_{9 / 2} \otimes v i_{13 / 2}$ band members in the even-even isotopes, and (iv) a comparison of the experimental $B(M 1) / B(E 2)$ ratios to theoretical calculations within the 3D-TAC model. For an independent check of the latter point we also calculated experimental $B(M 1) / B(E 2)$ values from our data set. For each level within the yrast band the intensities of the two decay branches, namely the $M 1$ and $E 2$ transitions, have been determined in coincidence with a $\gamma$-ray feeding the state to minimize systematic uncertainties. The result, which is shown in Fig. 7, is in agreement with Ref. [1] and therefore confirms the $\pi h_{9 / 2} \otimes v i_{13 / 2}$ assignment. However, because all the arguments for the assignment of $9^{-}$listed above are rather indirect ones, we prefer to consider this assignment and all the other ones based on it as preliminary. From the

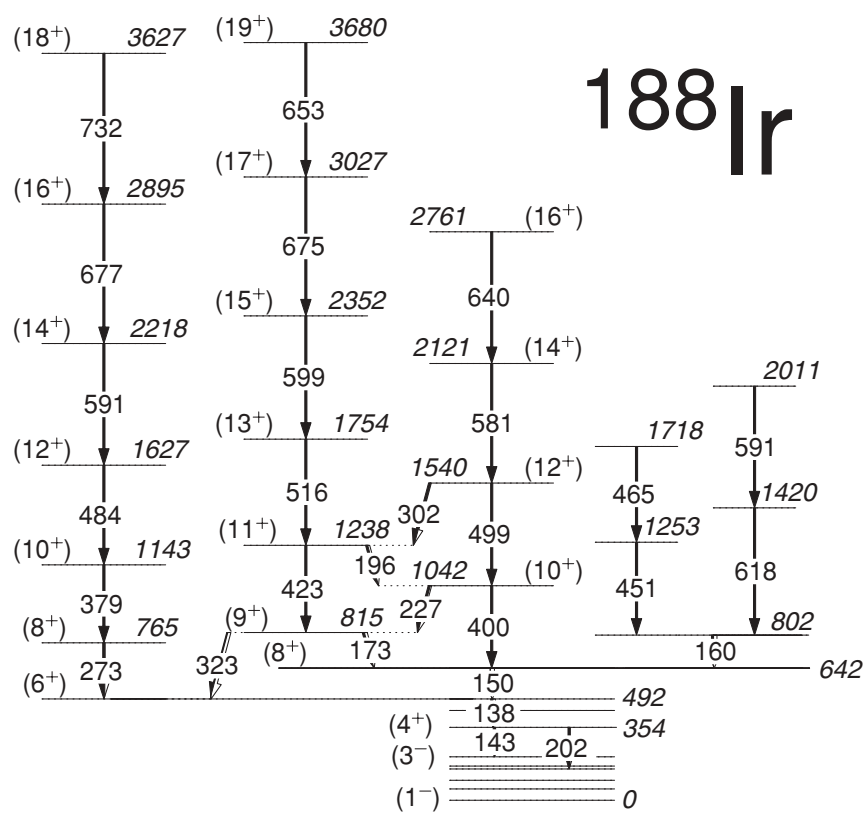

FIG. 6. Level scheme built on the ground state of ${ }^{188} \mathrm{Ir}$ as established in the present work.

observed intensity of the $88-\mathrm{keV}$ transition compared to the intensities of the two $\gamma$-rays of 633 and $266 \mathrm{keV}$ decaying from the $\Delta+1631-\mathrm{keV}$ state one can conclude that the $88-\mathrm{keV}$ transition has either $M 1$ or $E 2$ multipolarity. Taking into account the respective conversion coefficients, the assumption of $E 1, M 2$, or $E 3$ multipolarity would lead to a factor of 16,10 , and 25 , respectively, difference between incoming and outgoing intensities. This intensity argument limits the possible spin-parity/assignments to $\left(16^{-}, 17^{-}\right)$. Additional evidence for the assignment of $\left(16^{-}\right)$comes from the transition probabilities calculated from the measured lifetime of the $\Delta+1719-\mathrm{keV}$ level (see subsection II C) assuming different multipolarities. For an $E 2$ transition we obtain a transition probability of about 135 W.u., whereas for an $M 1$ transition a value around $2.6 \mathrm{~mW} . u$. is obtained.

The band head of band 2 was assigned $13^{-}$in Ref. [1] on the basis of its DCO ratio and the linear polarization measurement.

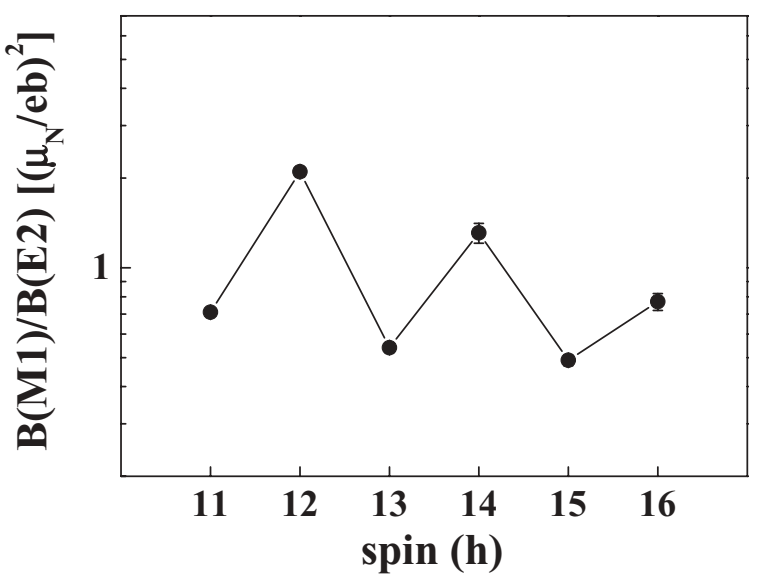

FIG. 7. Experimental $B(M 1) / B(E 2)$ ratios for the yrast band in 


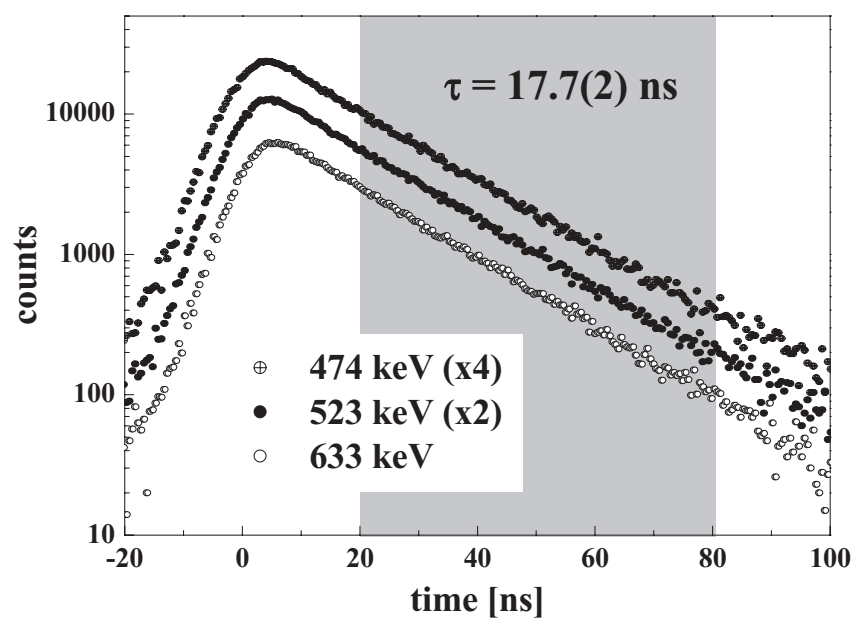

FIG. 8. Decay curves for the 474-, 523-, and 633-keV transitions in the decay cascade of the $\Delta+1719-\mathrm{keV}$ level in ${ }^{188} \mathrm{Ir}$.

This assignment is supported by the observed decay of this state and the $\left(14^{-}\right)$level of the same band to both the I-1 and I-2 states of band 1 .

\section{The lifetime of the $\Delta+1719-k e V$ state}

As mentioned above the $\left(16^{-}\right) \Delta+1719-\mathrm{keV}$ level had been identified to be an isomeric state on the basis of the observed $\gamma$-ray intensities of transitions above and below this state. To determine its lifetime, time spectra were produced for the delayed 633-, 523-, and 474-keV transitions in the main decay sequence of the isomer in coincidence with the prompt 575-keV transition above. Fits to the slopes of the decay curves shown in Fig. 8 in the region marked by a gray background yield lifetime values of 17.5(3), 17.6(3), and 18.0(3) ns, respectively. The resulting value for the lifetime of the $\left(16^{-}\right)$ isomeric state at $\Delta+1719 \mathrm{keV}$ is $\tau=17.7(2) \mathrm{ns}$.

\section{DISCUSSION}

The level schemes presented in Figs. 2 and 6, comprising regular rotational as well as irregular structures and isomeric states, clearly show that in the nucleus ${ }^{188} \mathrm{Ir}$ many different configurations with different properties, for example, with respect to deformation, compete. Soft heavy odd-odd nuclei in the middle of the shells are, in general, very difficult to describe in microscopic calculations with effective forces. To provide a theoretical analysis of at least some properties of ${ }^{188} \mathrm{Ir}$ we shall apply the Hartree-Fock-Bogoliubov theory within the Lipkin-Nogami approach with the finite-range density-dependent Gogny force (D1S parametrization). This approximation was developed in Ref. [10] for even-even nuclei and generalized for odd-even ones as to include exact blocking effects in Ref. [11]. In this work we apply it for the first time to the study of odd-odd systems. A very detailed description of the method can be found in Ref. [12]. In the present calculations we use a configuration space of 14 harmonic oscillator shells.

In a mean-field approach the blocking structure of the negative-parity states of an odd-odd nucleus is of the form $\pi^{+}, v^{-}$or $\pi^{-}, v^{+}$and $\pi^{+}, v^{+}$or $\pi^{-}, v^{-}$for the states of positive parity. In the particular case of the ${ }^{188} \mathrm{Ir}$ nucleus, for example, the $\pi^{+}, v^{-}$configuration means that we add to the even-even core ${ }^{186} \mathrm{Os}$ a proton in a positive-parity and a neutron in a negative-parity orbital. This is, of course, a simplified view because in a self-consistent approach as ours there is no core. Nevertheless it is convenient to look at the single-particle structure of the even-even core ${ }^{186} \mathrm{Os}$ to elucidate the orbitals relevant to the blocking structure in the proton and neutron channels of ${ }^{188} \mathrm{Ir}$. On the left-hand side of Fig. 9 the proton single particle levels of ${ }^{186}$ Os are displayed. Around the Fermi level and for small deformations the large angular momentum $h_{11 / 2}$ is the only negative-parity state available; at larger deformations the $h_{9 / 2}$ becomes also accessible. For the positive-parity states the small angular momentum $s_{1 / 2}$ and $d_{3 / 2}$ orbitals are the only candidates. As we can see on the right-hand side of Fig. 9, for neutrons we find the opposite situation. Around the Fermi level small angular momentum orbitals $\left(p_{1 / 2}, p_{3 / 2}\right.$, and $\left.f_{5 / 2}\right)$ are available in the negative-parity channel and the large angular momentum ones $\left(i_{13 / 2}\right.$ and $\left.g_{9 / 2}\right)$ at positive parity.

In the following we restrict ourselves to analyze the properties of the lowest-lying state of each of the four possible configurations $\left(\pi^{+}, v^{-}\right),\left(\pi^{-}, v^{+}\right),\left(\pi^{+}, v^{+}\right)$, and $\left(\pi^{-}, v^{-}\right)$in the nucleus ${ }^{188} \mathrm{Ir}$. Because this mass region is known to be soft against quadrupole deformations we shall investigate this dependence performing constrained Lipkin-Nogami HartreeFock-Bogoliubov (LNHFB) in the $\beta$ and $\gamma$ degrees of freedom. An important issue is the angular momentum of the nucleus. In our triaxial calculations this observable is not a conserved quantity. We could of course use the well-known semiclassical cranking technique to obtain spin conservation on the average. However, we do not find it appropriate for our purposes because, as mentioned, we are interested in the "ground state" of each of the four configurations and in these states there is no collective rotational angular momentum as generated by the cranking approach. Specifically, for odd-odd nuclei we minimize the LNHFB energy with constraints on the particle number and the $\beta$ and $\gamma$ deformations but we do not constrain on the angular momentum, which means the spins of the different isospin-parity channels are purely single particle. In Fig. 10 we show the potential energy contour plots; in the upper part for positive parity and in the lower part for negative parity. It is interesting to mention that the energy minima in the four channels are almost degenerated. As a matter of fact, the lowest energy corresponds to the $\left(\pi^{+}, v^{+}\right)$blocking structure, and the energy minima of the $\left(\pi^{+}, v^{-}\right),\left(\pi^{-}, v^{+}\right)$, and $\left(\pi^{-}, v^{-}\right)$ones are only 132,222 , and $271 \mathrm{keV}$ above, respectively. These minima are located at $\beta \approx 0.2$ and $\gamma \approx 30^{\circ}$ in all four channels. In these plots we can appreciate that the nucleus ${ }^{188} \mathrm{Ir}$ is extremely soft in the $\gamma$ degree of freedom. In all four isospin-parity channels the energy separation between prolate and oblate shapes is at most $0.5 \mathrm{MeV}$. The potential energy is also soft in the $\beta$ deformation parameter, energies corresponding to $\beta$ values of $0.2 \pm 0.05$ differ at most by $0.5 \mathrm{MeV}$.

Another important issue relevant to the blocking structure concerns the pairing correlations in the respective isospin-parity channels. Though in principle, due to the selfconsistency of the calculations, the pairing correlations of 


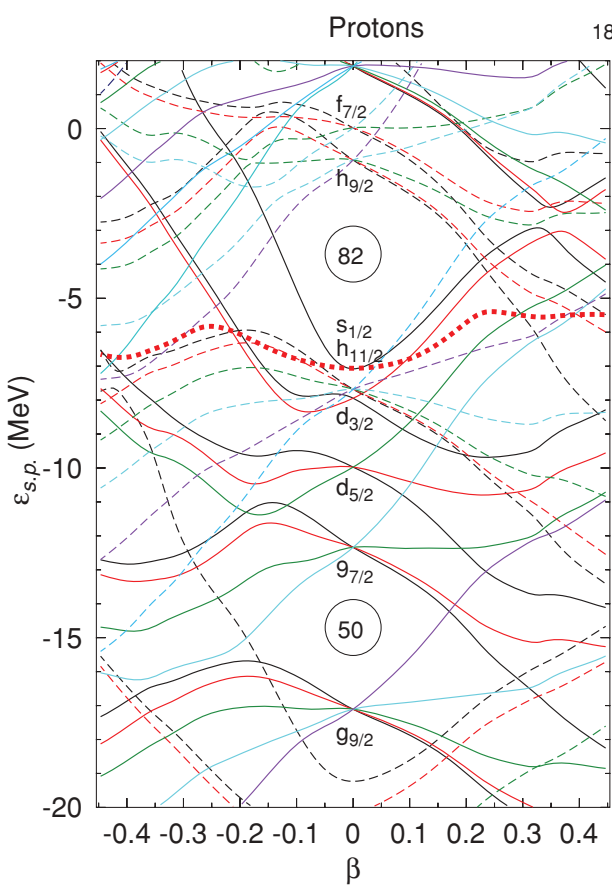

${ }^{186}$ Os

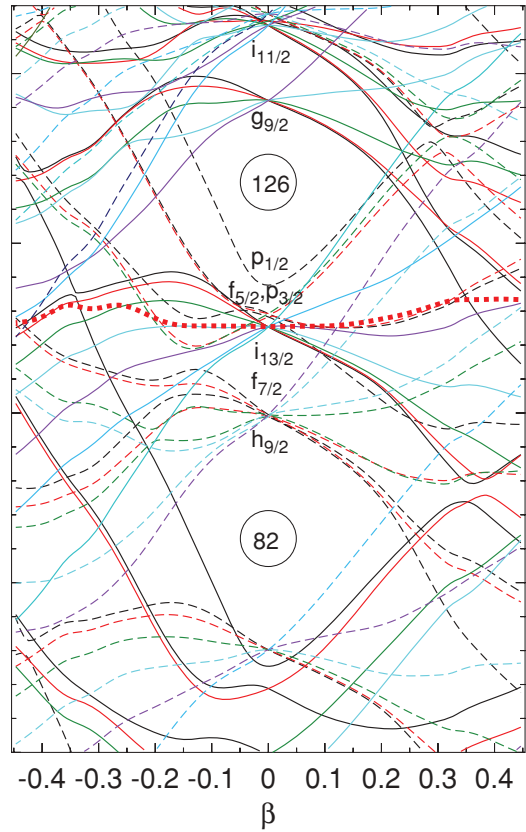

FIG. 9. (Color online) Single-particle energies of the nucleus ${ }^{186} \mathrm{Os}$, for protons (left) and neutrons (right) as a function of the deformation parameter $\beta$. Continuous (dashed) lines represent positive- (negative-) parity orbitals. The thick dashed lines represent the Fermi level. a given isospin-parity channel [say $E_{P}\left(\pi^{+}\right)$] depend on the parity of the other blocked particle (in this example if it is a $v^{+}$ or a $v^{-}$) in the actual calculations we observe only negligible differences and consequently we will only show them for one channel. In Fig. 11 we display the pairing correlations $-E_{P}$ defined by $E_{P}=-\frac{1}{2} \operatorname{Tr}\left(\Delta \kappa^{*}\right)$,with $\Delta_{k l}=\frac{1}{2} \sum_{m n} \bar{v}_{k l m n} \kappa_{m n}$ and $\kappa_{m n}=\left\langle\Phi\left|c_{n} c_{m}\right| \Phi\right\rangle$, with the trace and sums limited to an isospin-parity channel. These plots can be easily understood

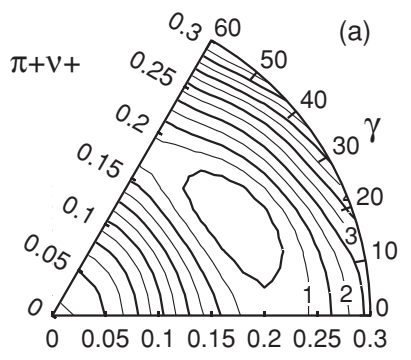

$\beta$

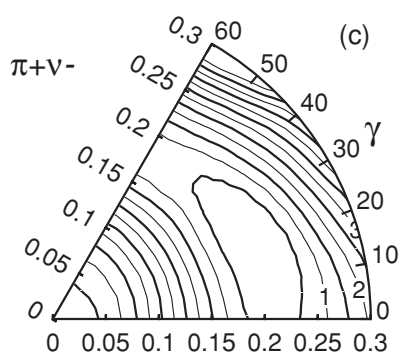

$\beta$

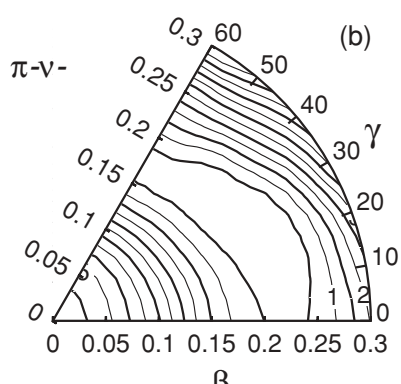

$\beta$

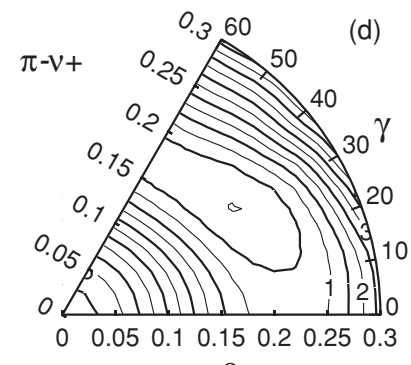

$\beta$
FIG. 10. Potential energy surface of the ${ }^{188}$ Ir nucleus in the $\beta-\gamma$ plane corresponding to the different configurations of isospin-parity blocking channels. In each channel the energy minimum has been set to zero. The contour lines are separated by $0.5 \mathrm{MeV}$. looking at Fig. 9 and from the consideration that pairing correlations are proportional to the level density. Around the Fermi levels we observe much larger level density for the neutron than for the proton system (compare Fig. 9), i.e., we expect larger pairing correlations for neutrons than for protons. The softening of the pairing correlations around $\beta=0.2$, especially for the proton system, can be understood
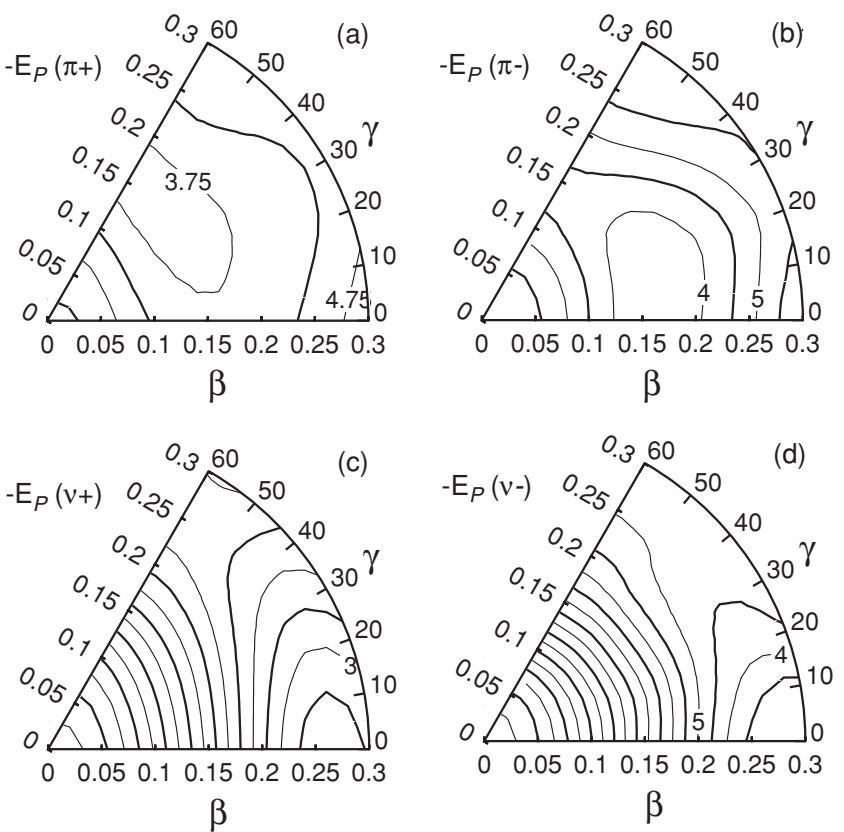

$\beta$

FIG. 11. Contour plot of the pairing energy correlations in the the $\beta-\gamma$ plane in the different isospin-parity channels. The contour lines are separated by $0.5 \mathrm{MeV}$. The pairing correlations at the origin are $E_{P}\left(\pi^{+}\right)=-5.25 \mathrm{MeV}, E_{P}\left(\pi^{-}\right)=-5.5 \mathrm{MeV}, E_{P}\left(v^{+}\right)=$ $-10 \mathrm{MeV}$, and $E_{P}\left(v^{-}\right)=-12 \mathrm{MeV}$. 
from Fig. 9 and from the observation that the binding energy minima are located at $\beta-\gamma$ deformation values with large shell gaps.

The large pairing correlations observed imply that there are several candidates to be the blocked states in each channel all of them lying close in energy, i.e., in each isopin-parity channel one has to take a linear combination of different blocked orbitals. All the results that have been presented up to now are clear indications that customary approximations used in detailed descriptions of nuclei will fail in this case. The large softness in the $\beta, \gamma$ degrees of freedom, see Fig. 10, indicates that one has to go beyond mean field, i.e., one has to use a kind of superposition of different shapes. For the same reason cranking techniques cannot be applied and one has to do exact triaxial angular momentum projection. These theories, known as the angular momentum projected generator coordinate method are now being developed for even-even nuclei $[13,14]$ but we are far from applying them to odd-odd nuclei.

Let us now look at the angular momentum contribution of the different channels, taking into account that in our calculations we impose the signature symmetry, $\left\langle J_{y}\right\rangle=\left\langle J_{z}\right\rangle=$ 0 and only $\left\langle J_{x}\right\rangle \neq 0$. Though, as before, the values of this quantity in a given isospin-parity channel in principle depend on the parity of the isospin partner, in the actual calculations this dependence is rather small and we shall discuss only the isolated channels. Taking into account the available orbitals around the Fermi level discussed above, it is clear that the blocking of a $\pi^{+}$(states available $s_{1 / 2}$ and $d_{3 / 2}$ ) or a $v^{-}$(states available $p_{1 / 2}, p_{3 / 2}, \ldots$ ) orbital does not provide a significant amount of angular momentum. However, the blocking of a $\pi^{-}$(states available $h_{11 / 2}$ and $h_{9 / 2}$ ) or a $v^{+}$ (states available $i_{13 / 2}$ and $g_{9 / 2}$ ) gives a substantial contribution to the spin. That means we expect large single-particle contributions to the angular momentum from the negativeparity configuration $\left(\pi^{-}, v^{+}\right)$, an intermediate one from the positive-parity configuration $\left(\pi^{-}, v^{-}\right)$and $\left(\pi^{+}, v^{+}\right)$and a small one from the negative-parity configuration $\left(\pi^{+}, v^{-}\right)$. In Fig. 12 we show the expectation value of $J_{x}$ for the $\pi^{-}$ and $v^{+}$isospin-parity channels. In the proton panel we find the largest contribution along the oblate axis and for medium $\beta$ values. In the neutron channel we also find large values along the oblate axis though they extend more to triaxial shapes. If we add both contributions we find a value of $9 \hbar$ for oblate shapes around $\beta=0.2$, which means, within our theoretical limitations, the most probable candidate for the observed $9^{-}$isomeric state is the $\left(\pi^{-}, v^{+}\right)$configuration. The contour plots for the expectation values of $J_{x}$ for the $\pi^{+}$and $v^{-}$configurations are flat and show small values in magnitude (typically $0-1 \hbar$ ) in the $\beta-\gamma$ plane. That means the present calculations indicate a $1^{-}$ground state of the type $\pi^{+}, v^{-}$ with $\beta \approx 0.2$ and the $\gamma$ value not well defined. Concerning
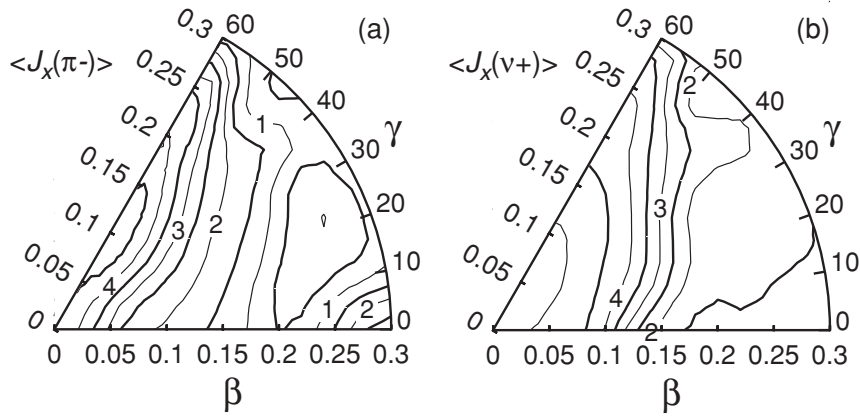

FIG. 12. Contour plot of the angular momentum in the $\beta-\gamma$ plane corresponding to the different isospin-parity blocking channels. The contour lines are separated by $0.5 \hbar$.

the $8^{+}$state, the angular momentum that we obtain from the $\left(\pi^{+}, v^{+}\right)$and $\left(\pi^{-}, v^{-}\right)$configurations is around $5 \hbar$ for an oblate configuration with $\beta \approx 0.2$. However, if one considers that the four configurations are very close in energy and that the $8^{+}$state lies $642 \mathrm{keV}$ above the ground state the missing $3 \hbar$ could be easily obtained by changing the occupation of an orbital with large angular momentum content. Obviously all these considerations are somewhat speculative.

\section{CONCLUSIONS}

To conclude, we presented extensive new experimental information about the level scheme of the odd-odd nucleus ${ }^{188} \mathrm{Ir}$. The recently proposed level structures built on the known $\mathrm{ms}$ isomeric state were revised and new rotational bands built on known low-spin states below the ms isomer were established. The basic features of the level scheme of ${ }^{188}$ Ir were analyzed within the Hartree-Fock-Bogoliubov theory within the Lipkin-Nogami appraoch with the finite-range densitydependent Gogny force. In light of the new experimental information, in particular the extension of the band proposed as chiral partner band ("Band 2" in Ref. [1]) of the yrast sequence in Ref. [1] and its decay properties, the assignment of these bands as first example of chiral twin bands in the $A \approx 190$ region appears doubtful.

\section{ACKNOWLEDGMENTS}

The authors acknowledge financial support from the spanish Ministerio de Educación y Ciencia within the programa Ramón y Cajal and research projects FPA2005-00696 and FIS2004-06697. We thank Professor Herbert Hübel from the University of Bonn for providing the ${ }^{186} \mathrm{~W}$ target.
[1] D. L. Balabanski, M. Danchev, D. J. Hartley, L. L. Riedinger, O. Zeidan, Jing-ye Zhang, C. J. Barton, C. W. Beausang, M. A. Caprio, R. F. Casten, J. R. Cooper, A. A. Hecht, R. Krücken, J. R. Novak, N. V. Zamfir, and K. E. Zyromski, Phys. Rev. C 70, 044305 (2004).
[2] C. Vaman, D. B. Fossan, T. Koike, K. Starosta, I. Y. Lee, and A. O. Macchiavelli, Phys. Rev. Lett. 92, 032501 (2004).

[3] K. Starosta, T. Koike, C. J. Chiara, D. B. Fossan, D. R. LaFosse, A. A. Hecht, C. W. Beausang, M. A. Caprio, J. R. Cooper, R. Krücken, J. R. Nowak, N. V. Zamfir, K. E. Zyromski, D. J. 
Hartley, D. Balabanski, Jing-ye Zhang, S. Frauendorf, and V. I. Dimitrov, Phys. Rev. Lett. 86, 971 (2001).

[4] J. Timár, C. Vaman, K. Starosta, D. B. Fossan, T. Koike, D. Sohler, I. Y. Lee, and A. O. Macchiavelli, Phys. Rev. C 73, 011301(R) (2006).

[5] V. I. Dimitrov, S. Frauendorf, and F. Dönau, Phys. Rev. Lett. 84, 5732 (2000).

[6] A. J. Kreiner, C. Alonso Arias, M. Debray, D. Digregorio, and A. Pacheco, Nucl. Phys. A425, 397 (1984).

[7] S. André, J. Boutet, J. Rivier, J. Treherne, J. Jastrzebski, J. Lukasiak, Z. Zujkowski, and C. Selville-Schuck, Nucl. Phys. A243, 229 (1975).
[8] P. Kemnitz, L. Funke, H. Sodan, E. Will, and G. Winter, Nucl. Phys. A245, 221 (1975).

[9] V. Modamio et al. (to be published).

[10] A. Valor, J. L. Egido, and L. M. Robledo, Phys. Lett. B392, 249 (1997).

[11] A. Villafranca and J. L. Egido, Phys. Lett. B408, 35 (1997).

[12] A. Valor, J. L. Egido, and L. M. Robledo, Nucl. Phys. A665, 46 (2000).

[13] R. Rodríguez-Guzmán, J. L. Egido, and L. M. Robledo, Nucl. Phys. A709, 201 (2002).

[14] T. R. Rodríguez and J. L. Egido, Phys. Rev. Lett. 99, 062501 (2007). 